\title{
Theorien, Methoden und Domänen der Folk Linguistics im deutschsprachigen Raum
}

\section{Einleitung in den Band}

Im deutschen Sprachraum hat sich seit den 1990er Jahren die Dialektologie verstärkt mit laienlinguistischen Vorstellungen zu deutschen Dialekten und zu deren Sprecherinnen und Sprechern befasst, anfangs noch unter Rückgriff auf verschiedene Termini (Alltagsdialektologie, Ethnodialektologie, Hörerdialektologie etc.). Mittlerweile konnte sich weitestgehend der Vorschlag von Anders (2010) durchsetzen, die diese Disziplin als Wahrnehmungsdialektologie bezeichnet (als Übersetzung des engl. perceptual dialectology). Eine Vielzahl von Publikationen ist hier zu Wissensinhalten und Methodologie erschienen (vgl. für eine Übersicht Hundt 2018). Doch damit ein umfassendes Verständnis von Sprachwandelprozessen, dem Entstehen von Normen und einem Konzept von Sprache insgesamt entstehen kann, ist nicht nur der Blick auf die Dialekte notwendig, sondern auf andere sprachliche Varietäten und damit verbunden auf einzelne Sprachsystemebenen. Diese Herangehensweise hat jedoch bisher in der Linguistik kaum Beachtung gefunden. Zwar hat sich schon Antos (1996) mit einer „Laienlinguistik“ beschäftigt, führt jedoch Untersuchungen von Sprachratgebern durch und versteht die Disziplin somit als eine Disziplin für Laien ${ }^{1}$. Dass jedoch auch eine Linguistik von Laien notwendig ist, wurde z. B. von Niedzielski \& Preston (2003) und Lehr (2002) gezeigt. Diese ist erst in den letzten Jahren im Entstehen begriffen wie bspw. das Handbuch von Antos, Niehr \& Spitzmüller (2019) verdeutlicht. Eine umfassende und insbesondere systematische Eruierung der laienlinguistischen Wissensbestände und Spracheinstellungen stellen ein dringendes Forschungsdesiderat dar. Dieser Sammelband, der im Anschluss an die Tagung LaienWissenSprache, die vom 5.-7. September 2019 an der Christian-Albrechts-Universität zu Kiel stattgefunden hat, möchte einen Beitrag zur Schließung der Forschungslücke leisten, aber auch neue Perspektiven für eine weiterführende Forschung eröffnen, die u. E. dringend geboten ist.

Diese Einleitung besteht aus insgesamt vier Teilen: Im ersten Abschnitt werden zentrale Erkenntnisinteressen der Laienlinguistik überblicksartig zu-

1 Die Varianten Laie und Experte verbleiben im typisierenden Singular, vgl. dazu die Fußnote im Beitrag Jürgen Spitzmüllers. 
sammengefasst. Der zweite Abschnitt beschäftigt sich mit der Frage nach der wissenschaftlichen Relevanz dieser Disziplin. Anschließend wird in Abschnitt 3 die Zielsetzung des Bandes dargestellt und im vierten Abschnitt wird ein Überblick über die Struktur dieses Bandes gegeben.

\section{Zentrale Erkenntnisinteressen der Laienlinguistik}

Die zentralen Erkenntnisinteressen der Laienlinguistik bzw. folk linguistics werden von Niedzielski \& Preston (2003: VII, Herv. i. Orig.) wie folgt formuliert:

In Folk Linguistics we hope to have shown that beliefs about, reactions to, and comments on language by what we call "real people" (i.e., nonlinguists) are interesting, illuminating, and empowering from ethnographic, linguistic, and practical (or applied linguistic) point of view.

Daraus lassen sich für die deutschsprachige Laienlinguistik zusammenfassend drei große Themenbereiche festhalten: 1. Sprachwissen, 2. Spracheinstellungen und 3. Sprachnormen. Diese sollen im Folgenden kurz näher erläutert werden.

Sprachwissen: Die Modellierung des Begriffs Sprachwissen ist vielschichtig und in der Forschung v. a. aus wissenssoziologischer Perspektive diskutiert worden (vgl. z. B. Anacker 2004; Beuge 2019; Berger \& Luckmann 1977/1969; Hoffmeister 2020a; Hundt 2017; Knoblauch 2014; Konerding 2015; Polanyi 1985; Schützeichel 2007; Schröder 2019; Strauss 2018). Dabei spielen unterschiedliche Perspektivierungen auf den Wissensbegriff eine Rolle. Zum einen ist die traditionelle Unterscheidung zwischen deklarativem und prozeduralem Wissen auch bei der Rekonstruktion laienlinguistischer Konzeptualisierungen relevant. So wird die Sprachkompetenz von Laien untersucht. Ein Verständnis laikaler Sprachkompetenz ist für verschiedene Forschungsbereiche grundlegend, da so Maßnahmen zur Regulation dieser Kompetenz ergriffen (Didaktik) und sprachliche Phänomene, wie z. B. Sprachwandelprozesse, erklärt werden können.

Zum anderen spielt - gerade beim laienlinguistischen Sprachwissen - die Differenzierung zwischen bewusst zugänglichem (explizierbarem) und unbewusst-intuitivem Wissen eine große Rolle. In jüngsten Erhebungen (vgl. Hundt 2017) zeigte sich u.a., dass linguistische Laien über verschiedene Wissensschichten (und Zugänglichkeitsgrade zu diesem Wissen) verfügen. Gerade die Fähigkeit linguistischer Laien, erst in einem allmählichen Prozess auf die eigenen Wissensbestände zuzugreifen, ist für die Rekonstruktion laienlinguistischer 
Konzeptualisierungen insgesamt relevant. Die damit verbundenen methodischen Probleme (valide und reliable Wissensrekonstruktion mittels geeigneter Erhebungsverfahren) sind ein nach wie vor bestehendes Forschungsdesiderat. Dies gilt z. B. auch für die kognitiven Prozesse, die bei der Bewältigung sprachlicher Problemfälle (Grammatikalitätsurteile, Beurteilung sprachlicher Zweifelsfälle, Einschätzungen zu salienten Sprachmerkmalen etc.) ablaufen (vgl. aber Hoffmeister 2020b). Schließlich geht es darum, den epistemischen Status von Laienwissen über Sprache im Wechselverhältnis zum sog. Expertenwissen zu klären, da sich beide Wissensbestände z. T. erheblich voneinander unterscheiden (vgl. Antos 1996: 163-164). So halten Welte \& Rosemann (1990: 1) fest:

Denn das alltägliche Meinen ,weiß‘, wie die Sprache entstanden ist, ,kennt‘ die ,erste‘ aller Sprachen und die ,beste‘. Es hat keinen Zweifel über die Grammatikalität von Sätzen und kann genau ,begründen', warum ein bestimmter Sprachgebrauch ,fehlerhaft" ist; es hat eine eindeutige Interpretation diachroner Veränderungen bei der Hand: als ,Sprachverfall‘.

Damit zielt die Modellierung sprachlichen Wissens zwischen Laien- und Fachwissen letztlich auch darauf ab, stereotype Vorstellungen zu sprachlichen Phänomenen zu dekonstruieren und ihr Zustandekommen zu erklären. Damit greift die Wissensrekonstruktion deutlich über rein innersprachliche Faktoren hinaus, da zur Beschreibung und Erklärung dieser stereotypen Wissensbestandteile (z. B. Korrelation zwischen Dialekten und bestimmten Persönlichkeitsmerkmalen bzw. Lebenshaltungen, oder die Korrelation zwischen orthographischen Kompetenzen und Intelligenzzuschreibungen) kulturelle, soziale, wirtschaftliche oder historische Faktoren herangezogen werden müssen.

Spracheinstellungen: Die Erhebung von Spracheinstellungen verfolgt einen anderen Ansatz und ein anderes Ziel als die von Sprachwissen. Während Sprachwissen sich idealerweise in die dichotome Kategorie Wahrheit einordnen lässt, geht es bei den Spracheinstellungen um individuelle oder soziale Vorlieben in Bezug auf bestimmte sprachliche Phänomene (vgl. z. B. Cuonz \& Studler 2014; überblicksartig Soukup 2019). In Abgrenzung zu Einstellungen gegenüber regionalen Varietäten (i. d. R. Dialekten, vgl. dazu z. B. Hundt 2012 mit weiterer Literatur, Hundt 1992) sind insbesondere Fragestellungen zur (situationsbedingten) Verwendung der alten Rechtschreibregeln interessant (vgl. Absatz zur Sprachnormforschung). Doch auch Einstellungen zu sozialen und funktionalen Varietäten (Jugendsprache, diverse Fachsprachen) sollen in diesem Zusammenhang diskutiert werden (vgl. dazu die Erhebungen, die im Kontext des Leibniz-Instituts für deutsche Sprache entstanden sind, exemplarisch Adler \& Plewnia 2018, 2019, 2020). 
Sprachnormforschung: Die Sprachnormforschung ist in der deutschsprachigen Linguistik bestens etabliert (vgl. z. B. grundlegend Gloy 1975; Beuge 2014, 2019 mit weiterer Literatur; Hundt 2009). Für den Sprachnormenbegriff linguistischer Laien gilt dies jedoch nicht in gleicher Weise (exemplarisch z. B. Dovalil 2006; Hoffmeister 2020b; Langer \& Davies 2006). Sprachnormen sind nach Gloy (1975: 61) keine sprachlich fixierten Normen,

d.h. Normformulierungen, sondern soziale Normen, die - gleichgültig, ob formuliert oder nicht formuliert - den Umfang, die Auswahl, den spezifischen Gebrauch von Sprachmitteln in irgendeiner Hinsicht als verbindlich festlegen.

Die Charakterisierung von Sprachnormen als soziale Normen im Sinne von „Erwartenserwartungen“ (Luhmann 1987: 33), die nicht zwingend formuliert und schriftlich fixiert sein müssen, reicht allein noch nicht als Grund für die wissenschaftliche Betrachtung aus. Beuge (2014: 129) hält jedoch fest, dass „die Bewertung sprachlicher Variation nicht nur ein fester Bestandteil der Sprachpraxis linguistischer Laien und ein notwendiger Faktor sozialer Interaktion, sondern auch ein wichtiger Motor des Sprachwandels“ ist. Insofern bedarf es einer eingehenden Untersuchung dessen, was Laien unter ,gutem oder richtigem Deutsch'verstehen. Hierbei sind zum einen grammatische Phänomene (z. B. der vermeintliche Genitivschwund) von Interesse, zum anderen aber auch die normative Wahrnehmung von Varietäten (z. B. Jugendsprache, Sprache in sozialen Medien).

\section{Wissenschaftliche Relevanz der Laienlinguistik}

Die Relevanz der eben umrissenen Laienlinguistik als einer Linguistik von Laien wird im angloamerikanischen Raum wenig bezweifelt. Hoenigswald (1966: 20) formuliert die folgende, viel zitierte Zusammenfassung der Relevanz der folk linguistics:

We should be interested not only in (a) what goes on (language), but also in (b) how people react to what goes on (they are persuaded, they are put off, etc.) and in (c) what people say goes on (talk concerning language).

Das Argument, dass sich die Fachwissenschaft für die Meinung der Sprachverwenderinnen und Sprachverwender interessieren sollte, ist jedoch ohne eine Begründung aus der Sache heraus nicht einwandfrei schlüssig. Unstrittig dürfte es jedoch sein, dass die Linguistik als Fachwissenschaft eine mehr deskriptive 
denn normative Rolle in verschiedenen Sprachdiskursen einnimmt (bzw. einnehmen soll). Selbst Wörterbücher wie beispielsweise der Duden bilden etablierte und sich etablierende Sprachphänomene ab, statt sie deduktiv zu oktroyieren. Ortner \& Sitta (2003: 11-12) formulieren schließlich das folgende Argument:

Ein Fach, das sich nicht an den Fragen und Bedürfnissen einer außerfachlichen Öffentlichkeit orientiert, läuft Gefahr, nur noch Fragen zu beantworten und Bedürfnisse zu erfüllen, die aus dem Fach selbst kommen. Mit anderen Worten: Es bietet Antworten an, wo Menschen außerhalb des Faches gar keine Fragen haben, es verliert damit seine Bodenhaftung, es gerät tendenziell zu einem elitären Zirkel. Je mehr es das tut, desto mehr verliert es an öffentlicher Reputation, es sinkt letztendlich zum Orchideenfach herab.

Doch auch für das inhaltliche Fortschreiten des Faches ist die Beschäftigung mit laienlinguistischen Fragestellungen nahezu systemimmanent. Die Sprachteilnehmerinnen und Sprachteilnehmer sehen sich dauerhaft sprachlichen Problemen und Zweifelsfällen (vgl. dazu Klein 2009, 2017; Schmitt, Szczepaniak \& Vieregge 2019) ausgesetzt, die eine unmittelbare Lösung erfordern. Hierbei bedarf es einer Sprachkompetenz, die zur Lösung derartiger Probleme unerlässlich ist. Erhebt die Linguistik an sich selbst nun den Anspruch, Inhalte allumfassend zu beschreiben, so ist die Beschäftigung mit denjenigen, die den Untersuchungsgegenstand verwenden und so für unmittelbar für den Wandel verantwortlich sind, eine Grundvoraussetzung (vgl. ausführlich Anders 2010: 22-26).

\section{Zielsetzung des Bandes}

Der vorliegende Band will in der deutschsprachigen Linguistik die Perspektive erweitern, hin zu einer breiten thematischen Vielfalt eben geschilderter Fragestellungen. Die Forschung zu laienlinguistischen Dialektkonzeptualisierungen ist mittlerweile auch im deutschen Sprachraum gut etabliert. Es liegt eine Reihe von einschlägigen Studien zu verschiedenen Dialekträumen vor (vgl. den Forschungsüberblick in Hundt 2018); das Forschungsgebiet ist darüber hinaus auch z. B. bei der Internationalen Gesellschaft für deutsche Dialektologie in Form einer eigenen Sektion vertreten (https://igdd.org/uber-die-igdd/sektionen/, letzter Zugriff 09.07.2020). Demgegenüber sind andere Varietäten der deutschen Sprache, d. h. situationale, mediale, funktionale etc. Erscheinungsformen der deutschen Sprache bislang kaum mit dem Fokus auf laienlinguistische Konzeptualisierungen untersucht worden. Zudem sind grundlegende theo- 
retische Konzepte der Laienlinguistik noch klärungsbedürftig (z. B. das Konzept des ,linguistischen Laien' selbst). Somit zeigen sich hier Forschungsdesiderate sowohl in theoretischer als auch in empirischer Hinsicht.

Aus diesem Grund setzt der Band verschiedene inhaltliche, theoretische, methodische und empirische Schwerpunkte.

Theorie/Methode/Empirie: Die eben genannten inhaltlichen Schwerpunkte werden jeweils unter den Aspekten der theoretischen Modellierung (z. B. das Konzept des linguistischen Laien, Struktur laienlinguistischer Konzepte) und der bei der Rekonstruktion linguistischen Laienwissens obwaltenden Erhebungsprobleme in methodischer Hinsicht diskutiert. Dies erfolgt - in einzelnen Beiträgen - am Beispiel von empirischen Untersuchungen zum sprachlichen Laienwissen.

Gegenstandsbereiche: Darüber hinaus geht es um die theoretische Grundlegung des Begriffs linguistischer Laie, der in den bisherigen Arbeiten eher vortheoretisch bzw. als gesetzt verwendet wurde. Zweitens wird es um die Modellierung laienlinguistischer Konzeptualisierungen selbst gehen. Dabei wird dann auch die Behandlung des vielschichtigen Wissensbegriffs (s. o.) eine Rolle spielen. Drittens sollen in verschiedenen Beiträgen Einstellungen zu verschiedenen Varietäten und Systembereichen der deutschen Sprache thematisiert werden.

Das übergeordnete Ziel des Bandes ist somit die Etablierung der folk linguistics/Laienlinguistik im Forschungskontext der germanistischen Sprachwissenschaft durch Beiträge von ausgewiesenen Expertinnen und Experten in diesem Bereich. Dass es in der germanistischen Sprachwissenschaft hier einen eklatanten Nachholbedarf im internationalen Vergleich gibt, haben die Ausführungen (Abschnitt 2) gezeigt.

\section{Die Struktur des Bandes}

Der Band ist in insgesamt vier übergeordnete Teile gegliedert, die ganz im Sinne der laienlinguistischen methodologischen Tradition das Resultat einer induktiven Kategorisierung der vielfältigen Beiträge darstellt. Wir kommen somit zu dem Ergebnis, dass die Studien laienlinguistisch interessierter Forscherinnen und Forscher vornehmlich folgende Bereiche fokussieren:

Teil 1: Theoretische Perspektiven

Teil 2: Geschichte und Gegenwart

Teil 3: Variation im Raum

Teil 4: Kommunikationsdomänen 
Der erste Beitrag aus dem Bereich Theoretische Perspektiven „His Master's Voice. Die soziale Konstruktion des ,Laien“ durch den ,Experten““ ist von Jürgen Spitzmüller und widmet sich der Frage, wie das Konzept LAIE durch den Experten konstruiert wird. Der Autor kommt dabei zu dem Ergebnis, dass das Verhältnis von Laien und Experten in der Sprachwissenschaft vor allem von einer Degradierung des Laien als vermeintlich ,Unwissenden“ geprägt ist. Diese Asymmetrie wird zudem noch durch die unterschiedlichen Reaktionsmechanismen von Sprachwissenschaftlerinnen und Sprachwissenschaftlern verstärkt, indem z. B. auch die folk linguistics den linguistischen Laien zwar Wissen zugesteht und dieses Wissen theoretisch $\mathrm{zu}$ fassen versucht. Allerdings kommt Spitzmüller zu dem Schluss, dass auch derlei Theoreme lediglich ein Versuch darstellen zu erklären, ,was‘ linguistische Laien eigentlich wissen. Der Autor plädiert hingegen für eine Umkehr der Perspektive, die die Hinwendung zum Experten beinhaltet.

Gerd Antos stellt in seinem Beitrag „Ist der Laie der Dumme? Erosion der Experten-Laien-Dichotomie in der Ära medial inszenierter Betroffenheit“ die Frage, weshalb sich das Verhältnis von Experten und Laien zunehmend verschiebt. Darauf gibt Antos drei Antworten: 1. Innerhalb der Gesellschaft werden Laien als Experten (des Ipsoflexiven) akzeptiert. 2. Dem voraus geht eine Inszenierung der Laien als Experten. 3. Als besonders wirkungsvolles Moment bestimmt Antos dabei die Betroffenheitsinszenierung, die zu dem gewachsenen Status aufgrund der Zuschreibung von Sonderwissen führt. Anhand des Beispiels der Leichten Sprache werden die Gedanken exemplarisch verdeutlicht.

In seinem Beitrag skizziert Ekkehard Felder die strukturelle Dialogizität zwischen Experten und Laien und schreibt dieser eine Orientierungsfunktion innerhalb partizipativer Wissensgesellschaften, die sich durch ein hohes Maß an Spezialisierung auszeichnen, zu. Dabei fokussiert der Beitrag die Entstehung, d. h. die diskursive Aushandlung geteilten Wissens und stellt die Frage nach der transparenten und nachvollziehbaren Gestaltung der Faktizitätsherstellung für Akteurinnen und Akteure wie Rezipientinnen und Rezipienten. Schließlich verweist der Beitrag auf die Unterscheidung von Daten und Fakten und entwirft ein Modell idealtypischer Transformationen in Vermittlungsprozessen, indem fünf Vermittlungsmodi unterschieden werden.

Toke Hoffmeister entwirft in seinem Beitrag „Sprachkonzepte in der Öffentlichkeit. Kognitive Repräsentationen der deutschen Sprache“ ein theoretisches Modell der Laienlinguistik, das unterschiedliche Aspekte dieses bislang in theo- 
retischer Hinsicht wenig erarbeiteten Bereichs adressiert. Mit den fünf Basisprinzipien der kognitiven Laienlinguistik (symbolisches Prinzip, Erfahrungsprinzip, Kontextualisierungsprinzip, Kategorisierungsprinzip, Gebrauchsbasiertheitsprinzip) und mit der Modellierung laienlinguistischen Wissens in Form eines Epistemikons wird ein Weg eröffnet, sowohl den vortheoretisch unreflektierten Begriff des linguistischen Laien inhaltlich näher $\mathrm{zu}$ bestimmen und zu definieren als auch die einerseits heterogenen andererseits auch übereinstimmenden Wissensbestände linguistischer Laien $\mathrm{zu}$ erfassen (Epistemikon). Am Beispiel der laienlinguistischen Konzeptualisierung zur DEUTSCHEN SPRACHE wird diese Modellierung anhand von Aussagen von Gewährspersonen erprobt.

Dirk Westerkamp geht aus philosophischer Perspektive auf die alltagssprachlich sich überlappenden Konzepte MEINEN, GLAUBEN und WisSEN ein und skizziert in seinem Beitrag „Meinen, Glauben, Laienwissen“ die unterschiedlichen Gelingensbedingungen dieser Sprechakte. Anhand von sechs Thesen arbeitet Westerkamp die Eigenheiten laienlinguistischer Wissensbestände heraus und kann dabei zeigen, dass die Erkenntnis der Existenz eines laikalen Wissens auf die Arbeiten Cusanus‘ zurückgeht. Diese Art von Wissen unterliegt verschiedenen Modi, die sich mindestens auf ein Wissen-dass und Wissen-wie zurückführen lassen. Ein von Laien geäußertes Wissen-dass ist dabei nicht selbstverständlicherweise als wahres und begründetes Wissen $\mathrm{zu}$ verstehen: Vielmehr tritt durch die Verwendung des Ausdrucks ich weiß die alethische Differenz zutage, die letztlich den Unterschied zwischen einem Wissen-dass und einem Fürwahrhalten erklärt. Die sechs Thesen führen letztlich zur notwendigen Differenzierung von Wissen und Erkenntnis auf der einen Seite und Vorstellung und Erfahrung auf der anderen Seite.

Simon Kasper und Christoph Purschke kritisieren in ihrem Beitrag „Kennen, Können, Wissen. Zur Konstruktion von Expertise“ die wissenschaftlichen Konzepte, die sich hinter den Begriffen Laie und Experte verbergen. Sie plädieren für ein neues Verständnis von Laien- und Expertentum und schlagen vor, das (Tun)Können als Basis des (Aus)Kennens zu verstehen; das Kundtun(können) ergänzt schließlich ihr Konzept von Expertise, die in dieser Form ihren notwendigen Platz in der Wissensgesellschaft erhalten kann.

Der Beitrag von Michael Frase und Rüdiger Harnisch „Der Experte als ideologieverdächtiger Laie“ widmet sich dem Spannungsfeld zwischen Wissenschaft/wissenschaftlicher Expertise und Einflussnahme in der Politik und Öffentlichkeit durch Linguistinnen und Linguisten. Die genaue Analyse 
verschiedener Beispiele kann zeigen, dass die wissenschaftliche Expertise allein nicht ausreichen kann, um konkrete politische Forderungen zu legitimieren bzw. Normen zu dekretieren. Hier müssen immer wieder ideologische, normative und ethische Vorannahmen, die auch die linguistische Sprachkritik beeinflussen, offengelegt und hinterfragt werden.

Ludwig M. Eichinger behandelt in seinem Beitrag „Sprache aus Sicht des Ohrenzeugen oder citizen science. Die Bandbreite des Laienwissens“ die neu aufkommende Herangehensweise der Citizen Science und beschreibt das sich im Zuge dessen wandelnde Verhältnis von Forscherinnen und Forschern zu Laien, die nun am Forschungsprozess beteiligt werden. Da Citizen Science nunmehr auch in der geisteswissenschaftlichen Forschung anzukommen scheint, diskutiert Eichinger die Folgen für die wissenschaftliche Praxis einerseits sowie die Konsequenzen für die Wissenschaftsdisziplinen, die sich, so Eichinger, einem Austausch nicht verwehren dürften, sondern mit dazu beitragen müssten, die unterschiedlichen Herangehensweisen an ein Thema sichtbar zu machen.

Die zweite Kategorie Geschichte und Gegenwart wird von Verena Sauer und ihrem Beitrag „,weil Geschmack und Feinheit der Sitten sich gemeiniglich um den Thron zu versammeln pflegen'. Historische Spracheinstellungen in Adelungs Lehrgebäude der Deutschen Sprache (1782)“ eingeleitet. Der Beitrag verbindet Arbeitsweisen der Spracheinstellungsforschung mit denen der Framesemantik und zeigt anhand einer exemplarischen Analyse mit Belegen aus dem Deutschen Textarchiv und aus Adelung (1782), dass sich die Slots des Frames Hochdeutsch im 18. Jahrhundert als sehr ähnlich zu denen heutiger Spracheinstellungen erweisen.

Der Beitrag von Wolf Peter Klein „Was denken linguistische Laien über die (deutsche) Grammatik. Beobachtungen und Interpretationen anhand des öffentlichen Sprachgebrauchs“ fragt in einem ersten Schritt nach den laienlinguistischen Wissensbeständen zur deutschen Grammatik. Davon ausgehend schlägt der Autor ein graduierendes Modell zur Bestimmung dessen vor, was unter einem linguistischen Laien bzw. einem linguistischen Experten verstanden werden kann. Es zeigt sich, dass hier von einem Kontinuum zwischen zwei Polen ausgegangen werden muss, das von verschiedenen Faktoren gesteuert wird. Anhand von acht Basiskonzeptualisierungen wird dann aufgrund einer Korpusanalyse gezeigt, wie das laienlinguistische Wissen zur deutschen Grammatik strukturiert ist. 
Astrid Adler und Albrecht Plewnia präsentieren mit ihrem Beitrag „Was denken linguistische Laien über Sprache? Ergebnisse einer aktuellen Repräsentativerhebung zu Spracheinstellungen in Deutschland“ einen Ausschnitt aus der sog. Deutschland-Erhebung aus dem Jahr 2017 und fokussieren dabei die Meinungen der Laien zu den Bereichen Zustand der deutschen Sprache, sprachliche Veränderungen, Anglizismen sowie den Einfluss anderer Sprachen. Der Vergleich mit den Ergebnissen weiterer Studien zeigt auf inhaltlicher Ebene, dass Vorstellungen zur deutschen Sprache insbesondere Negativ-Diskurse bedienen. Er zeigt auf methodischer Ebene aber auch eine deutlich erkennbare Abhängigkeit der Ergebnisse von der jeweils zugrundeliegenden Frageformulierung.

Der dritte Teil dieses Bandes Variation im Raum wird von Alfred Lameli und Gerhard Riener eingeleitet. Die Autoren gehen in ihrem Beitrag „Perzeption und Reaktion. Zum Einfluss regionaler Akzente auf das individuelle Handeln und dem Unterschied zwischen Bewertung und Verhalten“ der Frage nach, wie sich die Selbsteinschätzung von Probandinnen und Probanden in Abhängigkeit von präsentierten Sprechproben (z. B. Bairisch vs. Standardsprache) in Bezug auf kognitive Leistungstests verändert. Dazu werten die Autoren die Ergebnisse eines matched-guise-Experiments aus, bei dem thüringische Probandinnen und Probanden sich selbst und die (vermeintliche) Kontrahentin bzw. den (vermeintlichen) Kontrahenten einschätzen sollten. Je nach präsentiertem Hörproben (z. B. Bairisch vs. Standardsprache) unterscheiden sich die Selbsteinschätzungen deutlich. Bei regionalsprachlichen Stimuli überschätzen die thüringischen Probandinnen und Probanden ihre eigene Leistungsfähigkeit und unterschätzen die der (vermeintlichen) Kontrahentin bzw. des (vermeintlichen) Kontrahenten im Test. Dieser Effekt ist bei standardsprachlichen Stimuli nicht zu beobachten.

In ihrem Beitrag „Subjektiv-objektsprachliche Spektren im Raum“ gehen Brigitte Ganswindt, Juliane Limper und Lars Vorberger der Frage nach, welches Verhältnis subjektive und objektive Daten haben und wie dieses Verhältnis adäquat bestimmt werden kann. Zentral für die Studie ist die Bezeichnung der Sprachräume sowie die Konzeptualisierung des eigenen Sprachverhaltens (Autostereotyp). Das Ergebnis ist einerseits, dass es regionale Unterschiede in den Konzeptualisierungen gibt und andererseits dass die Kontrastierung subjektiver und objektiver Daten viel Potenzial besitzt und neue Erkenntnisse liefern kann.

In der Deutschschweiz, so Helen Christen und Alexandra Schiesser in ihrem Beitrag „Das Land, wo die Laien Dialektexperten sind“, würde über Sprache 
oftmals im Alltag reflektiert. Dies sei auf die polydialektale bzw. -glottale Situation zurückzuführen. Dieses so entstehende linguistische Laienwissen ist der Untersuchungsgegenstand der Autorinnen. Sie diskutieren anhand von Beispielanalysen der Radio-Talksendung Persönlich sowie von Dialekthematisierungen im Kontext des Forschungsprojektes Länderen ${ }^{n}$, wie oft Dialekt thematisiert wird, wenn er nicht explizit im Zentrum der Gesprächssituation steht bzw. welche Charakteristika die laienlinguistischen Konzeptualisierungen besitzen. Die Gegenüberstellung der beiden Kontexte (spontan in der Talk-Sendung bzw. geplant im Ländere ${ }^{n}$-Interview) ermöglicht so Differenzierungen des Laienwissens hinsichtlich der Granularität bzw. der Zugänglichkeitsgrade.

Der Beitrag „Linguistisches Laienwissen im mehrsprachigen Graubünden. Untersuchungsaufbau und Auswertungsmöglichkeiten“ von Noemi Adam-Graf fokussiert einen methodischen Aspekt und geht der Frage nach, ob bzw. inwieweit perzeptionslinguistische Methoden in einem mehrsprachigen Kontext anwendbar sind. Dabei wird anhand von Daten gezeigt, dass die etablierten Methoden (Fragebogenerhebung, semantisches Differential, Hand-Drawn-Maps) auch für ein mehrsprachiges Erhebungsumfeld geeignet sind.

Wolfgang Koppensteiner und Alexandra N. Lenz stellen in ihrem Beitrag „Standard(s) aus der Perspektive von „Nicht-LinguistInnen“ in Österreich“ Daten und Ergebnisse eines Forschungsprojekts im Rahmen des Spezialforschungsbereiches „Deutsch in Österreich. Variation - Kontakt - Perzeption“ (DiÖ) vor. Im Beitrag werden zwei Faktoren genauer untersucht. Zum einen die Rolle von Nachrichtensprecherinnen und Nachrichtensprechern und deren Sprachverhalten in Bezug auf die Konzeptualisierung von STANDARDSPRACHE durch linguistische Laien und zum anderen das laienlinguistische Konzept der (sprachlichen) REINHEIT. Es zeigt sich, dass „Nicht-LinguistInnen“ über differenzierte Konzeptualisierungen zur deutschen Standardsprache in Bezug auf deren Variationsbreite (vertikal) verfügen und dass dabei Nachrichtensprecherinnen und Nachrichtensprechern eine herausgehobene Funktion in der Frage nach dem sog. ,reinsten Deutsch` zugesprochen wird.

In ihrem Beitrag „Weil STANDARD verständlich ist und DIALEKT authentisch macht. Varietätenkonzeptionen im sekundären und tertiären Bildungsbereich in Österreich" stellen Philip C. Vergeiner, Elisabeth Buchner, Eva Fuchs und Stephan Elspaß die Fragen ins Zentrum, wie Sprecherinnen und Sprecher an Schule und Universitäten Dialekt und Standard konzeptualisieren, welche Bewertungen vorgenommen werden sowie welche normativen Ansprüche an die 
Sprache herangetragen werden. Der Beitrag zeigt, dass es zwischen den Konzeptualisierungen in Schule und Universität sowohl Gemeinsamkeiten als auch Unterschiede und Ambiguitäten sowie Widersprüche gibt, was die ohnehin komplexe sprachliche Situation in Österreich, wo Dialekt und Standard koexistieren, widerspiegelt.

Der vierte Abschnitt des Bandes widmet sich den Kommunikationsdomänen im laienlinguistischen Kontext und wird von Stephan Habscheid und Christine Hrncal eröffnet. Ihr Beitrag „Small Talk und Konversation“ verbindet gesprächslinguistische Analysen von Small-Talk-Kommunikation mit Vorschlägen zur Theoriebildung im Bereich kommunikativer Alltagspraktiken. Basis für die Analysen sind Pausengespräche im Theater, die im Rahmen eines DFG-Projekts („Theater im Gespräch“) erhoben wurden. Die Autorin und der Autor ermöglichen so einen neuen Blick auf die Gesprächssorte Small Talk, die - entgegen landläufiger Annahmen - sich nicht in der Umsetzung von beziehungs- und kontaktfunktionalen Aspekten erschöpft, sondern darüber hinaus bildungssprachliche Konversationsanteile transportiert. Die Autorin und der Autor zeigen ferner, dass und inwiefern dabei laienlinguistische Konzeptualierungen von Small Talk eine Rolle bei der konkreten Ausgestaltung dieser Gesprächssorte spielen.

Evelyn Ziegler gibt mit ihrem Beitrag „Wissenskonstruktion in Spracheinstellungsäußerungen zu Mehrsprachigkeit in der Linguistic Landscape der Metropole Ruhr" einen Einblick in die umfangreichen Ergebnisse des Forschungsprojekts „Metropolenzeichen“ (Kurztitel) und widmet sich der Frage, welche Argumentationsmuster den Spracheinstellungen zu visueller Mehrsprachigkeit in der Metropole Ruhr zugrunde liegen und damit Teil eines gesellschaftlichen oder subjektiven Wissensvorrats sind. Auf der Basis von 120 Interviews kann die Autorin zeigen, dass es vor allem soziale und räumliche Faktoren sind, die die Versprachlichungsstrategien der Gewährspersonen bestimmen. Inhaltlich lassen sich ihre Äußerungen jedoch größtenteils einem allgemeinen Wissensvorrat denn eigenen Erfahrungen zuschreiben.

Jörg Kilian fokussiert in seinem Beitrag „Didaktische Sprachkritik im Spannungsfeld zwischen Norm und Variation. Zur Markierung grammatischer Fehler in der Praxis der Bewertung von Schülerinnen- und Schülertexten im Deutschunterricht" das Problem der Bewertungen grammatischer Leistungen und Fehler von Schülerinnen und Schülern durch die Lehrkräfte. Es zeigt sich, dass sich die Bewertung standardsprachlicher Normen im Bereich der Grammatik aus der 
Sicht der sprachdidaktischen Sprachkritik anders darstellt als aus der Sicht der linguistischen Sprachkritik, weil dort z. T. andere Kriterien relevant sind. So ist es z. B. in manchen Fällen aus didaktischen Gründen geboten, standardsprachliche Variationsmöglichkeiten auszublenden. Lehrkräfte sind - dies zeigen die empirischen Analysen des Beitrags deutlich - eben nicht immer Sprachnormautoritäten im Sinne des Normenkonzepts von Ulrich Ammon.

Akra Chowchong behandelt in seinem Beitrag „,Don’t roll the r!“ - Metasprachdiskurse in Sprachlernvideos auf YouTube“ den Komplex der nichtinstitutionalisierten Deutschlernvideos. Im Zentrum stehen vor allem sprachideologische Positionierungen der Lehrenden sowie deren metasprachliche Kommentare. Gezeigt wird, dass eine hohe Normkonformität seitens der Lehrenden besteht, die starke Standardisierungstendenzen aufweisen. Variation wird demnach nicht thematisiert. Darüber hinaus erfolgt eine Autozuschreibung von Kompetenz durch die Rolle als Muttersprachlerin bzw. Muttersprachler. Der Beitrag verdeutlicht diese Aspekte in fünf beispielhaften Videoanalysen, die Aussprachevarianten von /r/ thematisieren.

Hannah Alker-Windbichler geht in ihrem Beitrag „Die Distanzierung von ,Krawallmädchen‘. Zur Rolle der Sprachwissenschaft in Debatten zur gendergerechten Sprache“ der Frage nach, welche Rolle der Sprachwissenschaft in Diskussionen in Online-Foren zur geschlechtergerechten Sprache beigemessen wird. Darüber hinaus untersucht der Beitrag, ob und welches Fachvokabular von den Diskutanten genutzt wird. Dabei wird gezeigt, dass die Disziplin der Linguistik als Autorität häufig zur Stärkung der eigenen Position herangezogen wird, auch wenn aktuelle Forschungsstände unberücksichtigt bleiben. Die linguistischen Themen sind darüber hinaus eng mit gesellschaftlichen Themen verknüpft, sodass das Bild der Sprachwissenschaft opak bleibt.

Der Beitrag „Die Partizipative Lücke im österreichischen Mandatsverfahren. Handlungswissen und Einstellungen bei Akteuren der Strafverfolgung“ von Daniel Leisser und Klara Kager widmet sich einem Problem der Angewandten Rechtslinguistik, bei dem das Spannungsverhältnis zwischen juristischen/linguistischen Laien einerseits und Experten andererseits relevant ist: das österreichische Mandatsverfahren, bei dem auf eine mündliche Verhandlung verzichtet wird. Die Autorin und der Autor stellen die Ergebnisse von fünf Experteninterviews vor, in denen die Wissensbestände, die Möglichkeiten und Probleme der Versprachlichung von rechtsbezogenen Konzepten und Prozessen thematisiert werden. 
Zum Gelingen der Tagung LaienWissenSprache, die vom 5.-7. September 2019 in Kiel stattgefunden hat und in deren Anschluss der vorliegende Band entstanden ist, wie auch zum Entstehen des Bandes haben zahlreiche Personen beigetragen: Zunächst danken wir allen Teilnehmerinnen und Teilnehmern der Tagung. Den Vorträgerinnen und Vorträgern, die die Laienlinguistik mit ihren Beiträgen vorangebracht haben aber auch den Zuhörerinnen und Zuhörern, die über die Diskussionsbeiträge wichtige Impulse geliefert haben. Außerdem bedanken wir uns bei Ulrike Zander, die mit exzellenter Vorbereitung und zuverlässiger Unterstützung den reibungslosen Ablauf der Tagung ermöglicht hat. Auch bei unseren Hilfskräften Luna Peters und Marit-Inga Suel bedanken wir uns herzlich für die Unterstützung während der Tagung.

Schließlich haben uns Luna Peters, Marrit Petzolt und Dinah Rohmann bei der Einrichtung des Bandes unterstützt. Dafür gilt ihnen unser herzlicher Dank. Dem Herausgeber der Reihe Sprache und Wissen, Ekkehard Felder, danken wir für die Aufnahme des Bandes in die renommierte Reihe. Außerdem danken wir Carolin Eckardt und Albina Töws vom de Gruyter-Verlag für die ausgezeichnete Betreuung sowie Gabriela Rus von Konvertus für die Unterstützung bei der Erstellung der Druckvorlage.

\section{Literaturverzeichnis}

Adler, Astrid \& Albrecht Plewnia (2018): Möglichkeiten und Grenzen der quantitativen Spracheinstellungsforschung. In Alexandra N. Lenz \& Albrecht Plewnia (Hrsg.), Variation - Normen - Identitäten, 63-97. Berlin, Boston: De Gruyter.

Adler, Astrid \& Albrecht Plewnia (2019): Die Macht der großen Zahlen. Aktuelle Spracheinstellungen in Deutschland. In Ludwig M. Eichinger \& Albrecht Plewnia (Hrsg.), Neues vom heutigen Deutsch. Empirisch - methodisch - theoretisch, 141-162. Berlin, Boston: De Gruyter.

Adler, Astrid \& Albrecht Plewnia (2020): Aktuelle Bewertungen regionaler Varietäten des Deutschen. Erste Ergebnisse des IDS-Sprachmoduls im SOEP-IS 2017. In Markus Hundt, Andrea Kleene, Albrecht Plewnia \& Verena Sauer (Hrsg.), Regiolekte. Objektive Sprachdaten und subjektive Wahrnehmung. Tübingen: Narr, Francke, Attempto.

Anacker, Michael (2004): Wissen. In Joachim Ritter, Karlfried Gründer \& Gottfried Gabriel (Hrsg.), Historisches Wörterbuch der Philosophie. Bd. 12, 891-900. Darmstadt: Wissenschaftliche Buchgesellschaft.

Anders, Christina. (2010): Wahrnehmungsdialektologie. Das Obersächsische im Alltagsverständnis von Laien. Berlin, New York: De Gruyter.

Antos, Gerd (1996): Laien-Linguistik. Studien zu Sprache- und Kommunikationsproblemen im Alltag. Am Beispiel von Sprachratgebern und Kommunikationstrainings. Tübingen: Niemeyer. 
Antos, Gerd, Thomas Niehr \& Jürgen Spitzmüller (Hrsg.) (2019): Handbuch Sprache im Urteil der Öffentlichkeit. Berlin, Boston: De Gruyter.

Berger, Peter L. \& Thomas Luckmann (1977/1969): Die gesellschaftliche Konstruktion der Wirklichkeit: Eine Theorie der Wissenssoziologie. Mit einer Einleitung zur deutschen Ausgabe von Helmuth Plessner. Übers. v. Monika Plessner. 5. Aufl. Frankfurt a. M.: Fischer.

Beuge, Patrick (2014): Was ist gutes Deutsch aus Sicht linguistischer Laien? Zeitschrift für Dialektologie und Linguistik 81 (2), 129-150.

Beuge, Patrick (2019): Was ist gutes Deutsch? Eine qualitative Analyse laienlinguistischen Sprachnormwissens. Berlin, Boston: De Gruyter.

Cuonz, Christina \& Rebekka Studler (Hrsg.) (2014): Sprechen über Sprache. Perspektiven und Methoden der Spracheinstellungsforschung. Tübingen: Stauffenburg.

Davies, Winifred (2010): Die Rolle (laien-)linguistischer Mythen bei der Reproduktion (sozio) linguistischer Normen. In Christina A. Anders, Markus Hundt \& Alexander Lasch (Hrsg.), „perceptual dialectology“. Neue Wege der Dialektologie, 385-408. Berlin, New York: De Gruyter.

Dovalil, Vit (2006): Sprachnormenwandel im geschriebenen Deutsch an der Schwelle zum 21. Jahrhundert. Die Entwicklung in ausgesuchten Bereichen der Grammatik. Frankfurt am Main u. a.: Lang.

Gessinger, Joachim (2008): Ethnodialektologie und sprachlicher Wandel. In Thomas Stehl (Hrsg.), Kenntnis und Wandel der Sprachen. Beiträge zur Potsdamer Ehrenpromotion für Helmut Lütke, 57-78. Tübingen: Narr.

Gloy, Klaus (1975): Sprachnormen I. Linguistische und soziologische Analysen. Stuttgart u. a.: Fromann-Holzboog.

Hoenigswald, Henry (1966): A proposal for the study of folk-linguistics. In William Bright (Hrsg.), Sociolinguistics, 16-26. The Hague: Mouton.

Hoffmeister, Toke (2020a): Die Aktivierung inaktiver Wissensbestände. Zur Repräsentation dialektologischen Wissens. In Markus Hundt, Andrea Kleene, Albrecht Plewnia \& Verena Sauer (Hrsg.), Regiolekte - objektive Sprachdaten und subjektive Wahrnehmung, 157-184. Tübingen: Narr.

Hoffmeister, Toke (2020b): Subjektive Grammatikalitätstheorien. Entstehung, Verbreitung und forschungspraktische Konsequenzen. Deutsche Sprache 3, 233-248.

Hundt, Markus (1992): Einstellungen gegenüber dialektal gefärbter Standardsprache. Eine empirische Untersuchung zum Bairischen, Hamburgische, Pfälzischen und Schwäbischen. Stuttgart: Steiner.

Hundt, Markus (2009): Normverletzungen und neue Normen. In Marek Konopka \& Bruno Strecker (Hrsg.), Deutsche Grammatik - Regeln, Normen, Sprachgebrauch, 117-140. Berlin, New York: De Gruyter.

Hundt, Markus (2012): Warum gibt es eigentlich „beliebte“ und „unbeliebte“ Dialekte? Theorien und Methoden der Einstellungsforschung im Bereich der Wahrnehmungsdialektologie. In Rainer Hünecke \& Karlheinz Jakob (Hrsg.), Die obersächsische Sprachlandschaft in Geschichte und Gegenwart, 175-222. Heidelberg: Winter.

Hundt, Markus (2017): Struktur und Komplexität des linguistischen Laienwissens. In Markus Hundt, Nicole Palliwoda \& Saskia Schröder (Hrsg.), Der deutsche Sprachrau aus der Sicht linguistischer Laien. Ergebnisse des Kieler DFG-Projektes, 121-160. Berlin, Boston: De Gruyter.

Hundt, Markus (2018): Wahrnehmungsdialektologie - Quo vadis? In Alexandra Lenz \& Albrecht Plewnia (Hrsg.), Variation - Norm(en) - Identität(en), 99-126. Berlin, Boston: De Gruyter. 
Hundt, Markus, Nicole Palliwoda \& Saskia Schröder (Hrsg.) (2017): Der deutsche Sprachraum aus der Sicht linguistischer Laien. Ergebnisse des Kieler DFG-Projektes. Berlin, Boston: De Gruyter.

Klein, Wolf Peter (2009): Auf der Kippe? Zweifelsfälle als Herausforderung(en) für Sprachwissenschaft und Sprachnormierung. In Marek Konopka \& Bruno Strecker (Hrsg.), Deutsche Grammatik - Regeln, Normen, Sprachgebrauch, 141-165. Berlin, New York: De Gruyter.

Klein, Wolf Peter (2017): Sprachliche Zweifelsfälle im Deutschen. Theorie, Praxis, Geschichte. Berlin, Boston: De Gruyter.

Knoblauch, Hubert (2014): Wissenssoziologie. 3., überarb. Aufl. Konstanz u. a.: UVK. Konerding, Klaus-Peter (2015): Sprache und Wissen. In Ekkehard Felder \& Andreas Gardt (Hrsg.), Handbuch Sprache und Wissen, 57-80. Berlin, Boston: De Gruyter.

Langer, Nils \& Winifred V. Davies (2006): The Making of Bad Language. Frankfurt am Main u. a.: Lang.

Lehr, Andrea (2002): Sprachbezogenes Wissen in der Lebenswelt des Alltags. Tübingen: Niemeyer.

Luhmann, Niklas (1987): Rechtssoziologie. 3. Aufl. Opladen: Westdeutscher Verlag.

Niedzielski, Nancy A. \& Dennis R. Preston (2003): Folk Linguistics. Berlin u. a.: Mouton de Gruyter.

Ortner, Hanspeter \& Horst Sitta (2003): Was ist der Gegenstand der Sprachwissenschaft? In Angelika Linke, Hanspeter Ortner \& Paul R. Portmann-Tselikas (Hrsg.), Sprache und mehr. Ansichten einer Linguistik der sprachlichen Praxis, 3-64. Tübingen: Niemeyer.

Polanyi, Michael (1985): Implizites Wissen. Frankfurt a. M.: Suhrkamp.

Preston, Dennis R. (1982): Perceptual dialectology. Mental maps of United States dialects from a Hawaiian perspective. Working Papers in Linguistics 14 (2), 5-49.

Preston, Dennis R. (1989): Perceptual dialectology: Nonlinguists' Views of Areal Linguistics. Dordrecht: Foris.

Preston, Dennis R. (1993): The uses of folk linguistics. International Journal of Applied Linguistics 3, 181-259.

Purschke, Christoph (2011): Regionalsprache und Hörerurteil: Grundzüge einer perzeptiven Variationslinguistik. Stuttgart: Steiner.

Schmitt, Eleonore, Renata Szczepaniak \& Annika Vieregge (Hrsg.) (2019): Sprachliche Zweifelsfälle. Definition, Erforschung, Implementierung. Hildesheim u. a.: Olms.

Schröder, Saskia (2019): Sprachräumliche Praxis. Sprachraumkartierung in der Wahrnehmungsdialektologie. Frankfurt am Main u. a.: Peter Lang.

Schützeichel, Rainer (Hrsg.) (2007): Handbuch Wissenssoziologie und Wissensforschung. Konstanz: UVK.

Soukup, Barbara (2019): Sprachreflexion und Kognition: Theorien und Methoden der Spracheinstellungsforschung. In Gerd Antos, Thomas Niehr \& Jürgen Spitzmüller (Hrsg.), Handbuch Sprache im Urteil der Öffentlichkeit, 83-106. Berlin, Boston: De Gruyter.

Strauss, Lina (2018): Rhetorikratgeber als Beispiel für Laienlinguistik. Eine Diskursanalyse. Berlin: Metzler.

Welte, Werner \& Philipp Rosemann (1990): Alltagssprachliche Metakommunikation im Englischen und Deutschen. Frankfurt a. M. u. a.: Lang. 